\title{
Homotopy Algorithm for Fixed Order Mixed $\mathrm{H}_{2} / \mathrm{H}_{\infty}$ Design M. Whorton, H. Buschek, A. J. Calise
}

Reprinted from

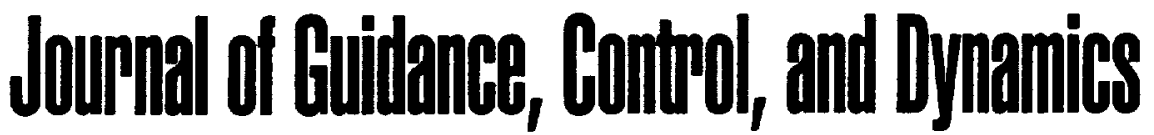

Volume 19, Number 6, Pages 1262-1269

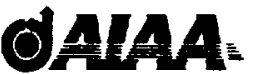




\title{
Homotopy Algorithm for Fixed Order Mixed $H_{2} / H_{\infty}$ Design
}

\author{
Mark Whorton* \\ NASA Marshall Space Flight Center, Huntsville, Alabama 35812 \\ and \\ Harald Buschek ${ }^{\dagger}$ and Anthony J. Calise ${ }^{\ddagger}$ \\ Georgia Institute of Technology, Atlanta, Georgia 30332
}

\begin{abstract}
Recent developments in the field of robust multivariable control have merged the theories of $\mathrm{H}_{\infty}$ and $\mathrm{H}_{2}$ control. This mixed $\boldsymbol{H}_{2} / \boldsymbol{H}_{\infty}$ compensator formulatton allows design for nominal performance by $\boldsymbol{H}_{2}$ norm minimization while guaranteeing robust stability to unstructured uncertainties by constraining the $H_{\infty}$ norm. A key dificulty associated with mixed $\boldsymbol{H}_{2} / \mathrm{H}_{\infty}$ compensation is compensator synthesis. A homolopy algorithm is presented for synthesis of fixed order mixed $\mathrm{H}_{2} / \mathrm{H}_{\infty}$ compensators. Numerical results are presented for a four disk flexible structure to evaluale the efficiency of the algorithm.
\end{abstract}

\section{Introduction}

$\mathbf{M}$ ODERN control theory has revolutionized control system design, with $\mathrm{H}_{2}$ and $\mathrm{H}_{\infty}$ methods gaining widespread recognition and application in controller synthesis for single-input/singleoutput (SISO) and multi-input/multi-output (MMO) problems. Early work in multivariable control synthesis utilized a quadratic cost functional to minimize the 2 norm of a system response to white noise inputs. Although the $\mathrm{H}_{2}$ procedure is well suited to many systems that specify performance in terms of rms quantities such as minimizing line-of-sight errors or control energy, it is well known that stability and performance cannot be guaranteed in the presence of model uncertainties. Robustness is addressed in $H_{\infty}$ control theory, which guarantees stability and performance (when defined by an $\infty$-norm measure) in the presence of unstructured uncertainty models, albeit often resulting in overly conservative designs. A significant disadvantage of these modern control techniques is that the resulting compensator is the same order as the generalized plant, which is often larger than the original plant due to the inclusion of frequency dependent weights to achieve the desired performance and robustness characteristics.

The consequential large controller order can be indirectly alleviated by reducing the order of the controller or alternatively by reducing the order of the design plant. In either case, indirect methods are suboptimal in performance and do not guarantee closed-loop stability. However, direct methods may be employed that impose constraints on controller order or architecture in the optimization procedure and, hence, provide stability and performance guarantees. In an optimization-based synthesis procedure, necessary conditions are formulated for the constrained closed-loop system that ensure internal stability. The optimal projection approach ${ }^{1}$ is an $H_{2}$ procedure whereby order constraints are imposed on the controller and the necessary conditions for minimizing a quadratic cost functional with respect to the fixed-order controller are derived. The resulting necessary conditions consist of two modified Riccati equations and two modified Lyapunov equations coupled by an oblique projection matrix. However, solution of the necessary conditions for realistic

\footnotetext{
Received March 26, 1996; revision received July 14, 1996; accepted for publication July 26,1996 . Copyright (C) 1996 by the American Institute of Aeronautics and Astronautics, Inc. No copyright is asserted in the United States under Titje 17. U.S. Code. The U.S. Government has a royalty-free license to exercise all rights under the copyright claimed herein for Governmental purposes. All other rights are reserved by the copyright owner.

*Aerospace Engineer, ED 12/Precision Pointing Control Systems. Member AIAA.

${ }^{\dagger}$ Graduate Research Assistant, School of Aerospace Engineering; currently Development Engineer, Missiles Division, FK-ESS, Bodenseewerk Gerätetechnik GmbH, P.O. Box 101155, D-88641 Überlingen, Germany. Member AIAA.

$\ddagger$ Professor, School of Aerospace Engineering. Fellow AIAA.
}

large-order systems is a difficult task. Homotopy methods have been employed to solve the optimal projection equations. ${ }^{2}$

As a means of providing robustness and performance, the mixed $\mathrm{H}_{2} / \mathrm{H}_{\infty}$ methodology has been developed. Much work has been done with variations of the mixed $H_{2} / H_{\infty}$ problem. (For a summary, see Ref. 3.) The earliest refereed work was done by Bernstein and Haddad, who extended the optimal projection approach to linear quadratic Gaussian (LQG) control with an $H_{\infty}$ norm constraint. Their formulation minimized an overbound on the $H_{2}$ norm from a disturbance input to one output while satisfying an $H_{\infty}$ norm overbound from the same disturbance input to a second output. The difficulty with this approach is the potential conservatism resulting from minimizing an overbound on the $H_{2}$ norm. The first attempt at solving the general mixed $H_{2} / H_{\infty}$ problem was by Rotea and Khargonekar, 5 who allowed independent inputs and outputs for the two transfer functions and minimized the actual $\mathrm{H}_{2}$ norm based on full state feedback. Ridgely and Walker ${ }^{3}$ extended the formulation to output feedback including the fixed-order case with either regular or singular $H_{\infty}$ constraints, and Canfield et at. ${ }^{6}$ provided a numerical solution. Another approach to the general mixed $\mathrm{H}_{2} / \mathrm{H}_{\infty}$ problem was developed by Sweriduk and Calise, ${ }^{7}$ who used a differential games formulation to obtain fixed-order controllers. A numerical solution of this formulation using homotopy was developed by Whorton et al., ${ }^{8}$ an extension of which is the subject of this paper. Davis et al. ${ }^{9}$ also recently developed an algorithm for mixed $H_{2} / H_{\infty}$ design.

Ridgely and Walker ${ }^{3}$ showed the mixed $H_{2} / H_{\infty}$ to be a strictly convex optimization problem with a unique solution when the controller is of the order equal to or larger than the underlying $\mathrm{H}_{2}$ problem. The solution is shown to lie on the boundary of the infinity norm constraint when active (for $\gamma<\bar{\gamma}$, where $\bar{\gamma}$ is the $H_{\infty}$ norm when the optimal $\mathrm{H}_{2}$ controller is used) and is just the $\mathrm{H}_{2}$ controller when $\gamma \geq \bar{\gamma}$. For controllers with order less than the underlying $\mathrm{H}_{2}$ problem, the possibility of local minima in the unconstrained $\mathrm{H}_{2}$ problem (optimal projection) exists and the solution of the mixed $\mathrm{H}_{2} / \mathrm{H}_{\infty}$ problem may or may not lie on the boundary of the $H_{\infty}$ norm constraint. As will be shown in the next section, Sweriduk and Calise? begin with the fixed-order $H_{\infty}$ cost functional and append the fixed-order $H_{2}$ cost functional. Reference 3 takes the converse approach by appending the $\mathrm{H}_{\infty}$ norm constraint to the $\mathrm{H}_{2}$ cost functional. As a consequence, the formulation in Ref. 3 can handle both regular and singular $H_{\infty}$ constraints and can be specialized to the $H_{2}$ problem. Conversely, the Ref. 7 formulation can handle both regular and singular $H_{2}$ constraints and can be specialized to the $H_{\infty}$ problem. However, the ability to handle singular constraints on either cost exists as long as one of the cost constraints is regular. Whereas the Ref. 7 formulation assumes that the plant dynamics ( $A$ matrix) of the two transfer functions are the same, Ref. 3 allows different dynamics in the two separate underlying $H_{\infty}$ and $H_{2}$ 
problems. Using the canonical compensator with a static gain output formulation presented in following section, the Ref. 7 formulation requires the simultaneous solution of five coupled nonlinear matrix equations. Reference 3 presents the necessary conditions in the form of seven coupled, nonlinear matrix equations.

The objective is to build on the results of Ref. 7 by presenting a homotopy algorithm that solves the mixed $\mathrm{H}_{2} / \mathrm{H}_{\infty}$ fixed-order compensator synthesis problem. Fixed-order $H_{2}$ and $H_{\infty}$ controllers are obtained as special cases of the algorithm. The paper is organized as follows. First, a formulation of the problem with the compensator in controller canonical form is presented and the necessary conditions for the fixed-order $\mathrm{H}_{2}$ controller are developed. These results are then extended to the fixed-order $H_{\infty}$ and mixed $H_{2} / H_{\infty}$ controller design using the differential game results of Ref. 7. (The approach and algorithms of this paper also follow Refs. 10 and 11 for $H_{2}$ controller synthesis.) Second, homotopy methods are introduced and a homotopy algorithm is developed to synthesize mixed $\mathrm{H}_{2} / \mathrm{H}_{\infty}$ compensators. Finally, numerical results are presented for evaluation of these homotopy algorithms followed by a discussion and concluding remarks.

\section{Problem Formulation}

The generalized plant of a standard control problem is given by

$$
\begin{gathered}
\dot{x}=A x+B_{1} w+B_{2} u \\
z=C_{1} x+D_{12} u \\
y=C_{2} x+D_{21} w+D_{22} u
\end{gathered}
$$

where $x \in R^{n}$ is the state vector, $w \in R^{n w}$ is the disturbance vector, $u \in R^{n u}$ is the control vector, $z \in R^{n z}$ is the performance vector, and $y \in R^{n y}$ is the measurement vector. The following is assumed.

1) $\left(A, B_{1}, C_{1}\right)$ is stabilizable and detectable.

2) $\left(A, B_{p}, C_{p}\right)$ is stabilizable and detectable.

3) $D_{12}$ has full column rank.

4) $D_{21}$ has full row rank.

A general compensator for this system is

$$
\begin{gathered}
\dot{\boldsymbol{x}}_{c}=A_{c} \boldsymbol{x}_{c}+B_{c} \boldsymbol{y} \\
u=C_{c} x_{c}
\end{gathered}
$$

where $x_{\mathrm{c}} \in R^{n c}$ is the state vector of the controller the dimension of which can be specified. Closing the loop using negative feedback yields the closed-loop system dynamics

$$
\begin{gathered}
\dot{\bar{x}}=\bar{A} \bar{x}+\tilde{B} w \\
z=\tilde{C} \bar{x}
\end{gathered}
$$

where

$$
\begin{gathered}
\overline{\boldsymbol{x}}=\left[\begin{array}{c}
\boldsymbol{x} \\
\boldsymbol{x}_{c}
\end{array}\right] \\
\tilde{A}=\left[\begin{array}{cc}
A & -B_{2} C_{c} \\
B_{c} C_{2} & A_{c}-B_{c} D_{22} C_{c}
\end{array}\right] \\
\tilde{B}=\left[\begin{array}{c}
B_{1} \\
B_{c} D_{21}
\end{array}\right] \\
\tilde{C}=\left[\begin{array}{ll}
C_{1} & -D_{12} C_{c}
\end{array}\right]
\end{gathered}
$$

The set of all internally stabilizing compensators is defined as

$$
S_{c}=\left\{\left(A_{c}, B_{c}, C_{c}\right): \tilde{A} \text { is asymptotically stable }\right\}
$$

For an $\mathrm{H}_{2}$ problem, the objective is to minimize the $\mathrm{H}_{2}$ norm on the closed-loop transfer function from disturbance inputs to performance outputs

$$
T_{z w}=\bar{C}(s I-\bar{A})^{-1} \tilde{B}
$$

where the disturbances are confined to the set of signals with bounded power and fixed spectra. If the disturbance is modeled as white noise, the objective is

$$
\min _{S_{c}}\left\{J\left(A_{c}, B_{c}, C_{c}\right)=\lim _{i \rightarrow \infty} E\left\{z(t)^{T} z(t)\right\}\right\}
$$

It can be shown that the cost can be expressed as

$$
J\left(A_{c}, B_{c}, C_{c}\right)=\operatorname{tr}\left\{Q \bar{B} \bar{B}^{T}\right\}=\operatorname{tr}\left\{P \bar{C}^{T} \tilde{C}\right\}
$$

where

$$
\begin{aligned}
& \bar{A} P+P \tilde{A}^{T}+\bar{B} \bar{B}^{T}=0 \\
& \tilde{A}^{T} Q+Q \bar{A}+\tilde{C}^{T} \bar{C}=0
\end{aligned}
$$

$P$ is the controllability grammian of $(\bar{A}, \tilde{B})$, and $Q$ is the observability grammian of $(\bar{C}, \vec{A})$. An equivalent cost functional also arises for the case of impulsive inputs.

To obtain the $H_{2}$ optimal compensator, the Lagrangian is defined as

$$
\mathcal{L}\left(Q, L, A_{c}, B_{c}, C_{c}\right)=\operatorname{tr}\left(Q \bar{B} \bar{B}^{T}+\left(\tilde{A}^{T} Q+Q \bar{A}+\bar{C}^{T} \tilde{C}\right) L\right\}
$$

where $L$ is a symmetric matrix of multipliers. Matrix gradients are taken to determine the first-order necessary conditions,

$$
\begin{gathered}
\frac{\partial \mathcal{L}}{\partial Q}=0, \quad \frac{\partial \mathcal{L}}{\partial L}=0, \quad \frac{\partial \mathcal{L}}{\partial A_{c}}=0 \\
\frac{\partial \mathcal{L}}{\partial B_{c}}=0, \quad \frac{\partial \mathcal{L}}{\partial C_{c}}=0
\end{gathered}
$$

Hence, computing an $\mathrm{H}_{2}$ optimal controller of fixed order $n c<n$ for the general controller structure given in Eqs. (4) and (5) requires the simultaneous solution of five coupled equations. This is not only computationally expensive but is also further complicated by the fact that the problem is overparametrized with such a compensator.

A controller form architecture ${ }^{12}$ is imposed on the compensator dynamics. This minimal realization avoids the problem of overparametrization and is a canonical form under mild conditions. ${ }^{13}$ The internal structure of the compensator is prespecified by assigning a set of feedback invariant indices $v_{i}$. In controller canonical form the compensator is defined as

$$
\begin{gathered}
\dot{\boldsymbol{x}}_{c}=P^{0} x_{c}+N^{0} u_{c}-N^{0} \boldsymbol{y} \\
u_{c}=-P x_{c} \\
u=-H x_{c}
\end{gathered}
$$

where $x_{c} \in R^{n c}$ and $u_{c} \in R^{n y}$. $P$ and $H$ are free-parameter matrices, and $P^{0}$ and $N^{0}$ are fixed matrices of zeros and ones determined by the choice of controllability indices. Similarly, a compensator in observer canonical form can be constructed. Only the controller canonical form is employed, which imposes the lower bound $n c \geq n y$ on the order of the compensator.

Let

$$
\bar{u}=\left[\begin{array}{l}
u \\
u_{c}
\end{array}\right]
$$

The augmented system may be expressed as

$$
\begin{aligned}
& \dot{\bar{x}}=\left[\begin{array}{cc}
A & 0 \\
-N^{0} C_{2} & P^{0}
\end{array}\right] \bar{x}+\left[\begin{array}{c}
B_{1} \\
-N^{0} D_{21}
\end{array}\right] w+\left[\begin{array}{cc}
B_{2} & 0 \\
-N^{0} D_{22} & N^{0}
\end{array}\right] \overline{\bar{u}} \\
& =\bar{A} \bar{x}+\bar{B}_{1} w+\bar{B}_{2} \bar{u} \\
& z=\left[\begin{array}{ll}
C_{1} & 0
\end{array}\right] \overline{\boldsymbol{x}}+\left[\begin{array}{ll}
D_{12} & 0
\end{array}\right] \bar{u} \\
& =\bar{C}_{1} \overline{\boldsymbol{x}}+\bar{D}_{12} \overline{\boldsymbol{u}}
\end{aligned}
$$




$$
\begin{gathered}
\bar{y}=\left[\begin{array}{ll}
0 & I
\end{array}\right] \overline{\mathrm{x}}=\bar{C}_{2} \bar{x} \\
\bar{u}=-\left[\begin{array}{l}
H \\
P
\end{array}\right] \bar{y}=-G \bar{y}
\end{gathered}
$$

Equations (24-27) define a static gain output feedback problem where the compensator is represented by a minimal number of free parameters in the design matrix $G$. The closed-loop system is given by

$$
\begin{aligned}
\dot{\bar{x}} & =\left(\bar{A}-\bar{B}_{2} G \bar{C}_{2}\right) \bar{x}+\bar{B}_{1} w \\
& =\tilde{A} \bar{x}+\bar{B} w \\
z & =\left(\bar{C}_{1}-\bar{D}_{12} G \bar{C}_{2}\right) \bar{x}=\bar{C} \bar{x}
\end{aligned}
$$

Minimizing the $H_{2}$ norm of $T_{z w}=\tilde{C}(s I-\tilde{A})^{-1} \tilde{B}$ utilizes the same Lagrangian as given in Eq. (18), but now $\mathcal{L}$ is only a function of three parameter matrices, i.e., $\mathcal{L}(Q, L, G)$. Thus, only three firstorder necessary conditions result:

$$
\begin{gathered}
\frac{\partial \mathcal{L}}{\partial Q}=\bar{A} L+L \bar{A}^{T}+\tilde{B} \bar{B}^{T}=0 \\
\frac{\partial \mathcal{L}}{\partial L}=\tilde{A}^{T} Q+Q \tilde{A}+\bar{C}^{T} \bar{C}=0 \\
\frac{\partial \mathcal{L}}{\partial G}=2\left(\bar{D}_{12}^{T} \bar{D}_{12} G \bar{C}_{2}-\bar{D}_{12}^{T} \bar{C}_{1}-\bar{B}_{2}^{T} Q\right) L \bar{C}_{2}^{T}=0
\end{gathered}
$$

Controller canonical forms can also be used to solve the $H_{\infty}$ problem. The objective is now to minimize the $\infty$ norm of the transfer function from disturbance inputs $w$ to performance outputs $z$ given in Eq. (13). In this case the necessary conditions for an $H_{\infty}$ suboptimal fixed-order compensator gain $G$ are

$$
\begin{aligned}
& \frac{\partial \mathcal{L}}{\partial Q_{\infty}}=\left(\bar{A}+\gamma^{-2} \bar{B} \bar{B}^{T} Q_{\infty}\right) L \\
& +L\left(\bar{A}+\gamma^{-2} \bar{B} \bar{B}^{T} Q_{\infty}\right)^{T}+\bar{B} \bar{B}^{T}=0 \\
& \frac{\partial \mathcal{L}}{\partial L}=\bar{A}^{T} Q_{\infty}+Q_{\infty} \bar{A}+\bar{C}^{T} \bar{C}+\gamma^{-2} Q_{\infty} \bar{B} \bar{B}^{T} Q_{\infty}=0 \\
& \quad \frac{\partial \mathcal{L}}{\partial G}=2\left(\bar{D}_{12}^{T} \bar{D}_{12} G \bar{C}_{2}-\bar{D}_{12}^{T} \bar{C}_{1}-\bar{B}_{2}^{T} Q_{\infty}\right) L \bar{C}_{2}^{T}=0
\end{aligned}
$$

where

$$
\begin{aligned}
& \mathcal{L}\left(Q_{\infty}, L, G\right)=\operatorname{tr}\left\{Q_{\infty} \tilde{B} \tilde{B}^{T}+\left(\tilde{A}^{T} Q_{\infty}+Q_{\infty} \tilde{A}\right.\right. \\
& \left.\left.\quad+\tilde{C}^{T} \bar{C}+\gamma^{-2} Q_{\infty} \tilde{B} \tilde{B}^{T} Q_{\infty}\right) L\right\}
\end{aligned}
$$

As in the $H_{2}$ problem, three coupled equations have to be solved to obtain a fixed-order compensator that satisfies the constraint $\left\|T_{z w}\right\|_{\infty}<\gamma$.

Fixed-order $H_{\infty}$ design has also been extended to fixed-order $\mu$ synthesis. ${ }^{14,15}$ Because $H_{\infty}$ controller design is a subproblem when designing for robust performance with structured uncertainty, the fixed-order technique just introduced has the potential to constrain the order of the controller that is normally subject to significant increases in the $\mu$-synthesis procedure.

The mixed $H_{2} / H_{\infty}$ problem can be approached in a similar fashion. ${ }^{7}$ In this case, the generalized plant has additional inputs and outputs $w_{p}$ and $z_{p}$, respectively, which define the $H_{2}$ performance criterion. The plant dynamics are illustrated in Fig. 1 . The inputs $w$ and outputs $z$ are used to define the $H_{\infty}$ performance criterion. Using the controller canonical form for the compensator, the augmented system for the mixed problem is

$$
\begin{gathered}
\dot{\bar{x}}=\bar{A} \bar{x}+\bar{B}_{1} w+\bar{B}_{p} w_{p}+\bar{B}_{2} \bar{u} \\
z_{p}=\bar{C}_{p} \bar{x}+\bar{D}_{1 p} \bar{u} \\
z=\bar{C}_{1} \bar{x}+\bar{D}_{12} \bar{u} \\
\bar{y}=\bar{C}_{2} \bar{x} \\
\bar{u}=-G \bar{y}
\end{gathered}
$$

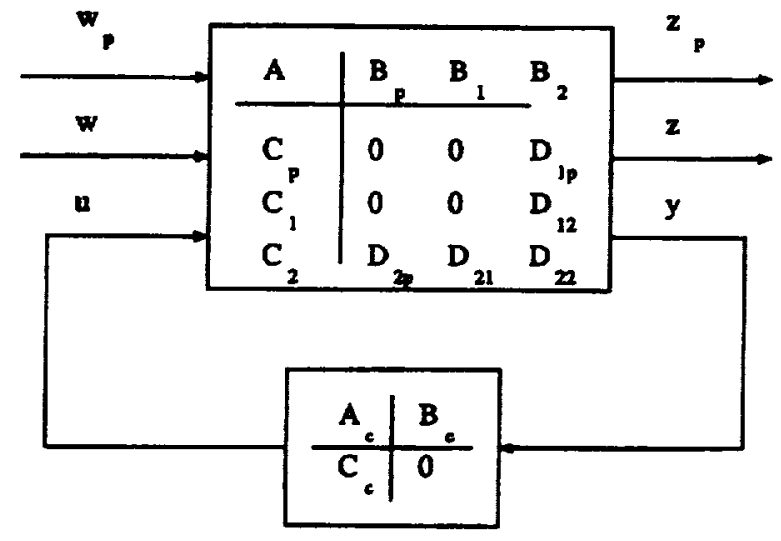

Fig. 1 Mbed $\boldsymbol{H}_{2} / \boldsymbol{H}_{\infty}$ problem.

where

$$
\begin{gathered}
\bar{B}_{p}=\left[\begin{array}{c}
B_{p} \\
-N^{0} D_{2 p}
\end{array}\right] \\
\bar{C}_{p}=\left[\begin{array}{ll}
C_{p} & 0
\end{array}\right] \\
\bar{D}_{1 p}=\left[\begin{array}{ll}
D_{1 p} & 0
\end{array}\right]
\end{gathered}
$$

The other expressions are the same as in Eqs. (24-27). Consequently, the closed-loop system is given by

$$
\begin{gathered}
\dot{\bar{x}}=\left(\bar{A}-\bar{B}_{2} G \bar{C}_{2}\right) \bar{x}+\bar{B}_{p} w_{p}+\bar{B}_{1} w \\
=\tilde{A} \bar{x}+\bar{B}_{p} w_{p}+\bar{B} w \\
z_{p}=\left(\bar{C}_{p}-\bar{D}_{1 p} G \bar{C}_{2}\right) \bar{x}=\bar{C}_{p} \bar{x} \\
z=\left(\bar{C}_{1}-\bar{D}_{12} G \bar{C}_{2}\right) \bar{x}=\bar{C} \bar{x}
\end{gathered}
$$

To formulate the performance index of the mixed problem, the Lagrangian for the $\mathrm{H}_{2}$ problem in Eq. (18) is adjoined to the Lagrangian for the $H_{\infty}$ problem in Eq. (36) by a scalar weight $\lambda$ :

$$
\begin{aligned}
\mathcal{L} & =\operatorname{tr}\left\{Q_{\infty} \tilde{B} \tilde{B}^{T}+\left(\tilde{A}^{T} Q_{\infty}+Q_{\infty} \tilde{A}\right.\right. \\
& \left.+\tilde{C}^{T} \tilde{C}+\gamma^{-2} Q_{\infty} \tilde{B} \tilde{B}^{T} Q_{\infty}\right) L+\lambda X \tilde{C}_{p}^{T} \tilde{C}_{p} \\
& \left.+\left(\bar{A} X+X \bar{A}^{T}+\tilde{B}_{p} \tilde{B}_{p}^{T}\right) L_{p}\right\}
\end{aligned}
$$

The weight $\lambda$ on the $H_{2}$ norm allows a tradeoff between performance ( $\mathrm{H}_{2}$ norm) and robustness $\left(H_{\infty}\right.$ norm). The first-order necessary conditions are

$$
\begin{aligned}
& \frac{\partial \mathcal{L}}{\partial Q_{\infty}}=\left(\bar{A}+\gamma^{-2} \tilde{B} \bar{B}^{T} Q_{\infty}\right) L \\
& +L\left(\tilde{A}+\gamma^{-2} \bar{B} \bar{B}^{T} Q_{\infty}\right)^{T}+\bar{B} \bar{B}^{T}=0 \\
& \frac{\partial \mathcal{L}}{\partial L}=\tilde{A}^{T} Q_{\infty}+Q_{\infty} \bar{A}+\tilde{C}^{T} \bar{C}+\gamma^{-2} Q_{\infty} \bar{B} \bar{B}^{T} Q_{\infty}=0 \\
& \frac{\partial \mathcal{L}}{\partial X}=\bar{A}^{T} L_{p}+L_{p} \tilde{A}+\lambda \tilde{C}_{p}^{T} \bar{C}_{p}=0 \\
& \frac{\partial \mathcal{L}}{\partial L_{p}}=\tilde{A} X+X \tilde{A}^{T}+\tilde{B}_{p} \bar{B}_{p}^{T}=0 \\
& \frac{\partial \mathcal{L}}{\partial G}=2\left[\bar{D}_{12}^{T} \bar{D}_{12} G \bar{C}_{2} L \bar{C}_{2}^{T}-\bar{D}_{12}^{T} \bar{C}_{1} L \bar{C}_{2}^{T}-\bar{B}_{2}^{T} Q_{\infty} L \bar{C}_{2}^{T}\right. \\
& \left.+\lambda \bar{D}_{1 p}^{T} \bar{D}_{1 p} G \bar{C}_{2} X \bar{C}_{2}^{T}-\lambda \bar{D}_{1 p}^{T} \bar{C}_{p} X \bar{C}_{2}^{T}-\bar{B}_{2}^{T} L_{p} X \bar{C}_{2}^{T}\right]=0
\end{aligned}
$$

Promising results have been obtained for the $H_{\infty}$ and the mixed problem where a conjugate gradient method was used in the 
computation. ${ }^{7}$ A disadvantage of this method is that convergence slows down near the optimum. Also, an initial starting guess for the compensator gain $G$ has to be provided that stabilizes the closedloop system. In this paper, a homotopy method is used to continuously deform the solution of a simple problem formulation to the solution of the desired problem formulation.

\section{Homotopy Methods}

Homotopy methods offer an attractive alternative to more standard approaches of optimal controller synthesis such as sequential and conjugate gradient methods. The basic philosophy of homotopy methods is to deform a problem that is relatively easily solved into the problem for which a solution is desired.

Homotopy (or continuation) methods, arising from algebraic and differential topology, embed a given problem in a parametrized family of problems. More specifically, consider sets $U$ and $Y \in \mathfrak{R}^{n}$ and a mapping $F: U \rightarrow Y$, where solutions of the problem

$$
F(u)=0
$$

are desired with $u \in U$ and $F(u) \in Y$. The homotopy function is defined by the mapping $H: U x[0,1] \rightarrow \mathfrak{R}^{n}$ such that

$$
H\left(u_{1}, 1\right)=F(u)
$$

and there exists a known (or easily calculated) solution $\boldsymbol{u}_{0}$, such that

$$
H\left(\boldsymbol{u}_{0}, 0\right)=0
$$

The homotopy function is a continuously differentiable function given by

$$
H[u(\alpha), \alpha]=0, \quad \forall \alpha \in[0,1]
$$

Thus, the homotopy begins with a simple problem with a known solution, Eq. (56), which is deformed by continuously varying the parameter until the solution of the original problem, Eq. (54), is obtained. ${ }^{16}$ The power of homotopy methods is that minimization is not strongly dependent on the starting solution but depends on local, small variations in the solution. Theoretically, these methods are globally convergent for a wide range of complex optimization problems, but in actuality, finite wordlength computation often introduces numerical ill-conditioning, resulting in difficulties with convergence. In light of these numerical limitations, a judicious choice of the initial problem and the associated initial stabilizing compensator is necessary for convergence and efficient computation. However, the ability to select an initial problem with a simple solution renders homotopy methods more widely applicable than sequential or gradient-based methods, which have a stringent requirement for an initial stabilizing solution.

Both discrete and continuous methods are used to sol ve the homotopy. Discrete methods simply partition the interval $[0,1]$ to obtain a finite chain of problems

$$
H\left(u, \alpha_{n}\right)=0, \quad 0=\alpha_{0}<\alpha_{1}<\cdots<\alpha_{N}=1
$$

Starting with a known solution at $\alpha_{n}$, the solution for $H\left(u, \alpha_{n+1}\right)$ is computed by a local iteration scheme. Continuous methods involve integration of Davidenko's differential equation, which is obtained by differentiating Eq. (57) with respect to $\alpha$, yielding

$$
\frac{\mathrm{d} u}{\mathrm{~d} \alpha}=-\left(\frac{\partial H}{\partial u}\right)^{-1} \frac{\partial H}{\partial \alpha}
$$

Given $u(0)=u_{0}$, this initial value problem may be numerically integrated to obtain the solution at $\alpha=1$ if the solution exists and is uniquely defined.

Research remains to be done in the application of homotopy algorithms. Efficient application of homotopy methods depends on the initial problem, the final problem, and the deformation undertaken. Given a good initial solution, the key to convergence is the ability to accurately track the solution curve, which is determined by the deformation undertaken. The ability to predict the solution along the homotopy path via Davidenko's differential equation makes continuous homotopy methods superior to discrete methods. Efficient computation of the Hessian is the primary issue for practical implementation of continuous homotopy algorithms. In the following sections, a continuous homotopy algorithm is presented for fixedorder mixed $\mathrm{H}_{2} / \mathrm{H}_{\infty}$ compensator design. The set of homotopy algorithms for synthesizing fixed-order $H_{2}, H_{\infty}$, and mixed $H_{2} / H_{\infty}$ compensators has been organized into a MATLAB ${ }^{\mathrm{TM}}$ toolbox called fixed-order compensation of uncertain systems (FOCUS)

\section{Homotopy Algorithm}

This section describes the algorithm used for implementing the continuous homotopy. In essence, a mixed discrete and continuous approach is employed where Davidenko's differential equation, Eq. (59), is integrated along the homotopy path, and at discrete points along the trajectory, a local optimization is used to remove integration error. The optimization scheme is a hybrid Newton/conjugate direction search method developed for this application. ${ }^{17}$ Local optimization at discrete points along the homotopy trajectory allows a crude integration procedure with large step sizes to be employed for efficiently tracking the solution curve. This approach follows closely that of Refs. 10 and 11 and is employed in the following algorithm.

1) Find initial solution $(\alpha=0)$.

2) Advance $\alpha$

$$
\alpha_{1, k}=\alpha_{0}+\Delta \alpha_{0, k}
$$

3) Predict $\theta$

$$
\theta\left(\alpha_{1, k}\right)=\theta\left(\alpha_{0}\right)+\Delta \alpha_{0, k} \theta^{\prime}\left(\alpha_{0}\right)
$$

where

$$
\theta^{\prime}(\alpha)=\frac{\mathrm{d} \theta}{\mathrm{d} \alpha}=-\left(\frac{\partial H}{\partial \theta}\right)^{-1} \frac{\partial H}{\partial \alpha}
$$

4) Check prediction error

$$
e_{k}(\theta, \alpha)=\left\|J_{\theta}\left[\theta\left(\alpha_{i, k}\right)\right]\right\|<\epsilon
$$

a) if error less than tolerance, continue; b) if not, $0.5 \Delta \alpha_{0 . k} \rightarrow$ $\Delta \alpha_{0, k+1} ;$ and $c$ ) increment $k$ and repeat steps $2-4$.

5) Correct with hybrid Newton/conjugate direction method to compute local minimum.

6) If $\alpha=1$, stop. Otherwise, go to step 2 .

Various approaches may be taken when selecting the deformation, but the general procedure applied in this effort is outlined as follows.

1) Synthesize a low-authority $\mathrm{H}_{2}$ (full-order) compensator.

2) Reduce the compensator to desired order and transform to canonical form. ${ }^{12}$

3) Set $\gamma$ large enough so that the $H_{2}$ and $H_{\infty}$ compensators are approximately equivalent.

4) Use homotopy to deform the initial low-authority, reducedorder $\mathrm{H}_{2}$ compensator into a full-authority reduced-order $\mathrm{H}_{2} \mathrm{com}$ pensator ( $\mathrm{H}_{2}$ homotopy).

5) Deform the full-authority $\mathrm{H}_{2}$ compensator into a nearly optimal $H_{\infty}$ compensator with $\gamma$ approaching its infimum ( $H_{\infty}$ homotopy).

6) At discrete values of $\lambda$, fix $\gamma$ and deform the compensator into the mixed $H_{2} / H_{\infty}$ compensator by varying $\lambda$ (mixed $H_{2} / H_{\infty}$ homotopy).

This procedure was chosen because it has been observed numerically that order reduction techniques tend to work best for lowauthority LQG controllers. ${ }^{10.18}$ A canonical form is imposed on the compensator structure to minimize the number of free parameters, which in some cases can also lead to numerical ill conditioning. A balancing transformation, which does not affect the controller characteristics, relaxes the strict structure in the $P^{0}$ and $N^{0}$ matrices in $\mathrm{Eq}$. (20) and improves the conditioning of the problem.

The procedure outlined separates the compensator synthesis into distinct phases. The initial reduced-order full-authority compensator is synthesized using the $H_{2}$ homotopy, which is then deformed into the reduced-order $H_{\infty}$ compensator. During the $H_{\infty}$ phase, the scalar $\mathrm{H}_{2}$ norm weight $\lambda$ is fixed (as are the plant matrices) and only the $H_{\infty}$ norm overbound $\gamma$ is varied. At discrete values, $\gamma$ is fixed and $\lambda$ is varied to perform the $\mathrm{H}_{2}$ norm minimization. Thus, the procedure alternates between the $H_{\infty}$ and $H_{2}$ norm minimization. 
During the homotopy, both the predicted and corrected gains are checked to ensure closed-loop stability. After each correction step, the cost gradient is checked to verify descent. During the $H_{\infty}$ homotopy, the solvability of the Riccati equation using predicted or corrected gains must also be checked. If any of these conditions are violated during correction, the correction step size is scaled and the condition is checked again. If scaling the correction step size is ineffective, the prediction step size is decreased and the prediction phase is repeated. This process continues until the homotopy is completed or until the prediction step size is decreased below a prespecified tolerance.

The following sections detail the derivations employed in the homotopy algorithm for mixed $\mathrm{H}_{2} / \mathrm{H}_{\infty}$ design. A complete development of the $H_{2}, H_{\infty}$, and mixed $H_{2} / H_{\infty}$ algorithms is given in Ref. 8.

\section{Mixed $\boldsymbol{H}_{2} / \boldsymbol{H}_{\infty}$ Development}

The homotopy function as well as the gradient and Hessian matrices are determined from the first-order necessary conditions for an optimal mixed $\mathrm{H}_{2} / \mathrm{H}_{\infty}$ compensator given by Eqs. (49-53). Define $\theta$ to be a vector comprising the free compensator parameters

$$
\theta=\operatorname{vec}(G)
$$

where $G$ is the output feedback gain matrix defined in Eq. (27). The gradient of the cost is

$$
f(\theta)=\frac{\partial \mathcal{L}}{\partial \theta}=\operatorname{vec}\left(\frac{\partial \mathcal{L}}{\partial G}\right)=0
$$

where $\partial \mathcal{L} / \partial G$ is given by Eq. (53).

The homotopy function is defined as

$$
H(\theta, \alpha)=\frac{\partial \mathcal{L}(\theta, \alpha)}{\partial \theta}=\operatorname{vec}\left(\frac{\partial \mathcal{L}(\theta, \alpha)}{\partial G}\right)=0
$$

Note that $\mathcal{L}$ is now a function of the homotopy parameter $\alpha$ since the system matrices are now functions of $\alpha$. The gradient of the homotopy function is

$$
\nabla\left[H^{T}(\theta, \alpha)\right]=\left[\begin{array}{ll}
\nabla_{\theta} H^{T} & \nabla_{\alpha} H^{T}
\end{array}\right]
$$

\section{Computation of Hessian}

The derivative of the $N \times 1$ vector valued homotopy function, $H^{T}(\theta)=\left[\boldsymbol{h}_{1}(\boldsymbol{\theta}), \boldsymbol{h}_{2}(\boldsymbol{\theta}), \ldots, \boldsymbol{h}_{N}(\theta)\right]$, with respect to the $N$ parameter vector $\theta$ is the $N \times N$ Hessian matrix given by

$$
\nabla_{\theta} H=\left[\begin{array}{llll}
\frac{\partial H}{\partial \theta_{1}} & \frac{\partial H}{\partial \theta_{2}} & \cdots & \frac{\partial H}{\partial \theta_{N}}
\end{array}\right]
$$

where

$$
\frac{\partial H}{\partial \theta_{j}}=\operatorname{vec}\left[\frac{\partial(\partial \mathcal{L} / \partial G)}{\partial \theta_{j}}\right]
$$

and using Eq. (53),

$$
\begin{aligned}
\frac{\partial H}{\partial \theta_{j}}= & \operatorname{vec}\left(\frac { \partial } { \partial \theta _ { j } } \left\{2 \left[\left(\bar{D}_{12}^{T} \bar{D}_{12} G \bar{C}_{2}-\bar{D}_{12}^{T} \bar{C}_{1}-\bar{B}_{2}^{T} Q_{\infty}\right) L \bar{C}_{2}^{T}\right.\right.\right. \\
& \left.\left.\left.+\left(\lambda \bar{D}_{1 p}^{T} \bar{D}_{1 p} G \bar{C}_{2}-\lambda \bar{D}_{1 p}^{T} \bar{C}_{p}-\bar{B}_{2}^{T} L_{p}\right) X \bar{C}_{2}^{T}\right]\right\}\right) \\
= & \operatorname{vec}\left\{2 \left[\left(\bar{D}_{12}^{T} \bar{D}_{12} G^{(j)} \bar{C}_{2}-\bar{B}_{2}^{T} Q_{\infty}^{(j)}\right) L \bar{C}_{2}^{T}\right.\right. \\
& +\left(\bar{D}_{12}^{T} \bar{D}_{12} G \bar{C}_{2}-\bar{D}_{12}^{T} \bar{C}_{1}-\bar{B}_{2}^{T} Q_{\infty}\right) L^{(j)} \bar{C}_{2}^{T} \\
& +\left(\lambda \bar{D}_{1 p}^{T} \bar{D}_{1 p} G{ }^{(j)} \bar{C}_{2}-\bar{B}_{2}^{T} L_{p}^{(j)}\right) X \bar{C}_{2}^{T} \\
& \left.\left.+\left(\lambda \bar{D}_{1 p}^{T} \bar{D}_{1 p} G \bar{C}_{2}-\lambda \bar{D}_{1 p}^{T} \bar{C}_{p}-\bar{B}_{2}^{T} L_{p}\right) X^{(j)} \bar{C}_{2}^{T}\right]\right\}
\end{aligned}
$$

The derivatives with respect to $\theta$ are denoted

$$
(*)^{(j)}=\frac{\partial(*)}{\partial \theta_{j}}
$$

For $\theta_{j}=g_{i k}$

$$
G^{(j)}=\frac{\partial G}{\partial \theta_{j}}=E_{i k}
$$

which is a matrix of zeros except for a one in the ik element.

To obtain expressions for $L^{(j)}, Q_{\infty}^{(j)}, L_{p}^{(j)}$, and $X^{(j)}$, differentiate Eqs. (49-52) with respect to $\theta_{j}$ to obtain

$$
\begin{aligned}
& 0=\left(\tilde{A}+\gamma^{-2} \tilde{B} \tilde{B}^{T} Q_{\infty}\right) L^{(j)}+L^{(j)}\left(\tilde{A}+\gamma^{-2} \bar{B} \bar{B}^{T} Q_{\infty}\right)^{T} \\
& +\left[\left(\bar{A}^{(j)}+\gamma^{-2} \tilde{B} \tilde{B}^{T} Q_{\infty}^{(j)}\right) L+L\left(\tilde{A}^{(j)}+\gamma^{-2} \tilde{B} \tilde{B}^{T} Q_{\infty}^{(j)}\right)^{T}\right] \\
& 0=\left(\bar{A}+\gamma^{-2} \bar{B} \bar{B}^{T} Q_{\infty}\right)^{T} Q_{\infty}^{(j)}+Q_{\infty}^{(j)}\left(\bar{A}+\gamma^{-2} \tilde{B} \tilde{B}^{T} Q_{\infty}\right) \\
& +\left[\bar{A}^{(j) \tau} Q_{\infty}+Q_{\infty} \bar{A}^{(j)}+\left(\tilde{C}^{\tau} \bar{C}\right)^{(j)}\right] \\
& 0=\bar{A}^{T} L_{p}^{(j)}+L_{p}^{(j)} \bar{A}+\left[\bar{A}^{(j) T} L_{p}+L_{p} \tilde{A}^{(j)}+\lambda\left(\tilde{C}_{p}^{T} \tilde{C}_{p}\right)^{(j)}\right] \\
& 0=\bar{A} X^{(j)}+X^{(j)} \bar{A}^{T}+\left[\tilde{A}^{(j)} X+X \bar{A}^{(j) T}+\left(\tilde{B}_{p} \bar{B}_{p}^{T}\right)^{(j)}\right]
\end{aligned}
$$

Derivatives of the closed-loop matrices are obtained from Eqs. (45-47) and are given by

$$
\tilde{A}^{(j)}=-\bar{B}_{2} G^{(j)} \bar{C}_{2}
$$

$$
\left(\tilde{B} \tilde{B}^{T}\right)^{(j)}=\left(\bar{B}_{p} \tilde{B}_{p}^{T}\right)^{(j)}=0
$$

$$
\begin{aligned}
& \left(\bar{C}^{T} \bar{C}\right)^{(j)}=\left(\bar{C}_{2}^{T} G^{(j) T} \bar{D}_{12}^{T} \bar{D}_{12} G \bar{C}_{2}\right)+\left(\bar{C}_{2}^{T} G^{(j) T} \bar{D}_{12}^{T} \bar{D}_{12} G \bar{C}_{2}\right)^{T} \\
& -\left(\bar{C}_{1}^{T} \bar{D}_{12} G^{(j)} \bar{C}_{2}\right)-\left(\bar{C}_{1}^{T} \bar{D}_{12} G^{(j)} \bar{C}_{2}\right)^{T} \\
& \left(\tilde{C}_{p}^{T} \tilde{C}_{p}\right)^{(j)}=\left(-\bar{D}_{1 p} G^{(j)} \bar{C}_{2}\right)^{T}\left(\bar{C}_{p}-\bar{D}_{1 p} G \bar{C}_{2}\right) \\
& +\left(\bar{C}_{p}-\bar{D}_{1 p} G \bar{C}_{2}\right)^{T}\left(-\bar{D}_{1 p} G^{(j)} \bar{C}_{2}\right)
\end{aligned}
$$

Computation of $H_{\alpha}$

Similarly, the derivative of the homotopy function with respect to the homotopy parameter $\alpha$ is the $N \times 1$ vector

$$
\nabla_{\alpha} H^{T}=\frac{\partial H^{T}}{\partial \alpha}
$$

and

$$
\begin{aligned}
\frac{\partial H}{\partial \alpha}= & \operatorname{vec}\left[\frac{\partial(\partial \mathcal{L} / \partial G)}{\partial \alpha}\right] \\
= & \operatorname{vec}\left(\frac { \partial } { \partial \alpha } \left\{2 \left[\left(\bar{D}_{12}^{T} \bar{D}_{12} G \bar{C}_{2}-\bar{D}_{12}^{T} \bar{C}_{1}-\bar{B}_{2}^{T} Q_{\infty}\right) L \bar{C}_{2}^{T}\right.\right.\right. \\
& \left.\left.\left.+\left(\lambda \bar{D}_{1 p}^{T} \bar{D}_{1 p} G \bar{C}_{2}-\lambda \bar{D}_{1 p}^{T} \bar{C}_{p}-\bar{B}_{2}^{T} L_{p}\right) X \bar{C}_{2}^{T}\right]\right\}\right) \\
= & \operatorname{vec}\left\{2 \left[\left(\dot{\bar{D}}_{12}^{T} \bar{D}_{12} G \bar{C}_{2}+\bar{D}_{12}^{T} \dot{\bar{D}}_{12} G \bar{C}_{2}-\dot{\bar{D}}_{12}^{T} \bar{C}_{1}\right.\right.\right. \\
& \left.-\bar{B}_{2}^{T} \dot{Q}_{\infty}\right) L \bar{C}_{2}^{T}+\left(\bar{D}_{12}^{T} \bar{D}_{12} G \bar{C}_{2}-\bar{D}_{12}^{T} \bar{C}_{1}-\bar{B}_{2}^{T} Q_{\infty}\right) \dot{L} \bar{C}_{2}^{T} \\
& \left.\left.+\left(\dot{\lambda} \bar{D}_{1 p}^{T} \bar{D}_{1 p} G \bar{C}_{2}-\dot{\lambda} \bar{D}_{1 p}^{T} \bar{C}_{p}-\bar{B}_{2}^{T} \dot{L}_{p}\right) X \bar{C}_{2}^{T}\right]\right\}
\end{aligned}
$$

where

$$
(\dot{*})=\frac{\partial(*)}{\partial \alpha}
$$


Implicit in these equations is the assumption that all system matrices are fixed with the exception of $\bar{D}_{12}$ and $\bar{D}_{1 p}$ and the parameters $\lambda$ and $\gamma$. In general, the homotopy can be performed with any/all system matrices deformed, but the methodology for mixed $\mathrm{H}_{2} / \mathrm{H}_{\infty}$ employed is to vary only one parameter at a time.

The derivative terms in the third equality in Eq. (79) depend on the deformation undertaken in the specified problem, i.e., the initial and final problem. Suppose that the deformation of the matrix $\bar{D}_{12}$ is prescribed to be

$$
\bar{D}_{12}(\alpha)=\bar{D}_{12,0}(\alpha)+\alpha\left[\bar{D}_{12, f}(\alpha)-\bar{D}_{12,0}(\alpha)\right]
$$

where the 0 and $f$ subscripts indicate the initial and final system matrices, respectively. It follows that

$$
\dot{\bar{D}}_{12}=\bar{D}_{12 . f}-\bar{D}_{12.0}
$$

The derivative of other plant matrices and the parameters $\lambda$ and $\gamma$ are determined accordingly. To obtain expressions for the derivatives of $L, Q_{\infty}, L_{p}$, and $X$ with respect to $\alpha$, Eqs. (49-52) are differentiated, resulting in the following:

$$
\begin{aligned}
0 & =\left(\tilde{A}+\gamma^{-2} \tilde{B} \tilde{B}^{T} Q_{\infty}\right) \dot{L}+\dot{L}\left(\tilde{A}+\gamma^{-2} \tilde{B} \tilde{B}^{T} Q_{\infty}\right)^{T} \\
& +\left(\Gamma L+L \Gamma^{T}\right) \\
0 & =\left(\tilde{A}+\gamma^{-2} \tilde{B} \tilde{B}^{T} Q_{\infty}\right)^{T} \dot{Q}_{\infty}+\dot{Q}_{\infty}\left(\tilde{A}+\gamma^{-2} \bar{B} \bar{B}^{T} Q_{\infty}\right) \\
& -\left(2 \gamma^{-3} \dot{\gamma} Q_{\infty} \tilde{B} \tilde{B}^{T} Q_{\infty}\right) \\
0= & \tilde{A}^{T} \dot{L}_{p}+\dot{L}_{p} \bar{A}+\left(\dot{\bar{A}}^{T} L_{p}+L_{p} \dot{\bar{A}}+\lambda \dot{\bar{C}}_{p}^{T} \tilde{C}_{p}\right. \\
& \left.+\lambda \bar{C}_{p}^{T} \dot{\bar{C}}_{p}+\dot{\lambda} \tilde{C}_{p}^{T} \tilde{C}_{p}\right) \\
& 0=\bar{A} \dot{X}+\dot{X} \tilde{A}^{T}+\left(\dot{\bar{A}} X+X \dot{\bar{A}}^{T}+\dot{\tilde{B}}_{p} \tilde{B}_{p}^{T}+\tilde{B}_{p} \dot{\bar{B}}_{p}^{T}\right)
\end{aligned}
$$

where

$$
\Gamma=\dot{\bar{A}}-2 \gamma^{-3} \dot{\gamma} \bar{B} \bar{B}^{T} Q_{\infty}+\gamma^{-2}\left(\dot{\bar{B}} \tilde{B}^{T} Q_{\infty}+\bar{B} \dot{\bar{B}}^{T} Q_{\infty}+\bar{B} \tilde{B}^{T} \dot{Q}_{\infty}\right)
$$

and, from Eqs. (45-47),

$$
\begin{gathered}
\dot{\bar{A}}=\dot{\bar{A}}-\dot{\bar{B}}_{2} G \bar{C}_{2}-\bar{B}_{2} G \dot{\bar{C}}_{2} \\
\dot{\bar{B}}_{p}=\dot{\bar{B}}_{p} \\
\dot{\vec{B}}=\dot{\bar{B}}_{1} \\
\dot{\bar{C}}_{p}=\dot{\bar{C}}_{p}-\dot{\bar{D}}_{1 p} G \bar{C}_{2}-\bar{D}_{1 p} G \dot{\bar{C}}_{2} \\
\dot{\bar{C}}=\dot{\bar{C}}_{1}-\dot{\bar{D}}_{12} G \bar{C}_{2}-\bar{D}_{12} G \dot{\bar{C}}_{2}
\end{gathered}
$$

The use of the canonical compensator formulation in the augmented system dynamics simplifies not only the necessary conditions but also the derivative expressions of the system matrices. From Eqs. (37-40), the augmented matrix derivatives reduce to

$$
\begin{gathered}
\dot{\bar{A}}=\left[\begin{array}{cc}
A_{f}-A_{0} & 0 \\
-N^{0}\left(C_{2, f}-C_{2,0}\right) & 0
\end{array}\right] \\
\dot{\bar{B}}_{p}=\left[\begin{array}{cc}
B_{p, f}-B_{p, 0} \\
-N^{0}\left(D_{2 p, f}-D_{2 p, 0}\right)
\end{array}\right] \\
\dot{\bar{B}}_{1}=\left[\begin{array}{cc}
B_{1, f}-B_{1,0} \\
-N^{0}\left(D_{21, f}-D_{21,0}\right)
\end{array}\right] \\
\dot{\bar{B}}_{2}=\left[\begin{array}{cc}
B_{2, f}-B_{2,0} & 0 \\
-N^{0}\left(D_{22, f}-D_{22,0}\right) & 0
\end{array}\right] \\
\dot{\bar{C}}_{p}=\left[\begin{array}{ll}
C_{p, f}-C_{p, 0} & 0
\end{array}\right]
\end{gathered}
$$

$$
\begin{gathered}
\dot{\bar{C}}_{1}=\left[\begin{array}{cc}
C_{1, f}-C_{1.0} & 0
\end{array}\right] \\
\dot{\bar{C}}_{2}=\left[\begin{array}{ll}
0 & 0
\end{array}\right] \\
\dot{\bar{D}}_{1 p}=\left[\begin{array}{ll}
D_{1 p . f}-D_{1 p .0} & 0
\end{array}\right] \\
\dot{\bar{D}}_{12}=\left[\begin{array}{ll}
D_{12 . f}-D_{12.0} & 0
\end{array}\right]
\end{gathered}
$$

The presence of the zero subblocks significantly enhances the computational efficiency of this approach. When implementing the procedure described at the beginning of this section, the preceding equations may be further specialized. In the initial $\mathrm{H}_{2}$ homotopy procedure the initial and final plant matrices are the same and the homotopy is performed only on the measurement and process noise intensities, $D_{12}$ and $D_{21}$. Hence $\dot{A}, \dot{B}_{2}, \dot{C}_{1}$, and $\dot{C}_{2}$ are identically zero. For the mixed $H_{2} / H_{\infty}$ homotopy, the $H_{\infty}$ and $H_{2}$ homotopies are performed distinctly, which simplifies the computations significantly because the plant matrices remain fixed and only $\gamma$ or $\lambda$ are varied at one time.

\section{Design Example}

To demonstrate the homotopy algorithm applied to optimal controller synthesis, the four disk example originally described in Ref. 19 and more recently by numerous others ${ }^{10}$ will be used. The four disk model used in the example problem was derived from a laboratory experiment and represents an apparatus developed for testing of pointing control systems for flexible space structures with noncollocated sensors and actuators. Four disks are rigidly attached to a flexible axial shaft with control torque applied to selected disks and the angular displacement of selected disks measured. The plant parameters are taken from Ref. 4.

\section{$\mathrm{H}_{2}$ Case}

To demonstrate the homotopy algorithm applied to $\mathrm{H}_{2}$ controller synthesis, a direct comparison between the homotopy algorithm of Ref. 10 called HAO and the FOCUS algorithm (called H2HOM for the $\mathrm{H}_{2}$ case) will be presented first. The main distinction between the two homotopy algorithms for the $H_{2}$ case is the compensator architecture. HAO employs a general architecture that may be restricted to various parametrizations including the controllability canonical form, which is similar to the controller canonical form used in H2HOM for this SISO example problem. The HAO code has been highly optimized for efficient computation with the result that superfiuous computations are not evaluated. The homotopy algorithm of this paper is patterned after the general approach of HAO and utilizes some of the more efficient computational aspects of the HAO code.

The control design philosophy for this example is to scale the nominal control weight and the nominal sensor noise intensity by the parameter $q$. As $q$ is reduced, the control authority is increased. For comparison with the published results in Ref. 10, a full- (eighth-) order compensator was synthesized. Although the results can be directly obtained from the LQG Riccati equations, the full-order compensator was chosen to tax the H2HOM algorithm, which must optimize over a greater number of parameters with increasing compensator order.

Table 1 shows a comparison of the results from the H2HOM and HAO algorithms for the full-order compensator along with results from the H2HOM algorithm for sixth- and second-order compensators. All pertinent parameters as well as logic for step-size scaling and the computation of the prediction and correction errors are identical in both algorithms, which are implemented in MATLAB on a $48666-\mathrm{MHz}$ computer. Whereas the HAO code required a minimum step size of $1.907 \mathrm{e}-7$, the H2HOM solution was much better conditioned and required a minimum step size of 0.025 . As a consequence of the smaller step sizes with HAO, 2504 Hessian computations were required, as opposed to only 63 Hessian computations with H2HOM. The HAO code has been tuned extensively for efficient computation, as is reflected in the small number of floating point operations per second (flops) required. In spite of the significantly smaller number of flops with HAO, the H2HOM code required significantly less clock time for convergence to the same final compensator. (The results generated by the authors using the 
Table 1 Comparison of H2HOM and EAO algorithms

\begin{tabular}{lcccc}
\hline \hline Algorithm & HAO & H2HOM & H2HOM & H2HOM \\
\hline $\begin{array}{l}\text { Compensator order } \\
\text { Number of Hessian }\end{array}$ & 8 & 8 & 6 & 2 \\
$\quad$ computations & 2504 & 63 & 60 & 30 \\
$\begin{array}{l}\text { Min. step size } \\
\text { Max. step size }\end{array}$ & $1.907 e-7$ & 0.025 & 0.05 & 0.1 \\
$\begin{array}{l}\text { Max. number of correction } \\
\text { iterations }\end{array}$ & 0.1 & 0.1 & 0.1 & 0.2 \\
Mflops & 9 & 7 & 8 & 5 \\
Time, s & 287 & 936 & 455 & 37 \\
\hline \hline
\end{tabular}

Table 2 Comparison of FOCUS and Ref. 4 results

\begin{tabular}{lllcc}
\hline \hline$\gamma$ & $\left\|T_{z w}\right\|_{\infty, \mathrm{BH}}$ & $\left\|T_{z w}\right\|_{\infty, F}$ & $\left\|T_{\text {zpwp }}\right\|_{2, \mathrm{BH}}$ & $\left\|T_{\text {zpwp }}\right\|_{2, F}$ \\
\hline 2.0 & 1.18 & - & 0.382 & - \\
1.5 & 1.06 & - & 0.389 & - \\
1.0 & 0.855 & 0.947 & 0.410 & 0.398 \\
0.9 & 0.797 & 0.889 & 0.420 & 0.403 \\
0.8 & 0.732 & 0.776 & 0.432 & 0.421 \\
0.7 & 0.661 & 0.668 & 0.451 & 0.438 \\
0.6 & - & 0.589 & $\overline{0.512}$ & 0.460 \\
0.52 & 0.511 & 0.520 & - & 0.508 \\
0.5 & - & 0.5 & - & 0.518 \\
0.4 & - & 0.398 & - & 0.572 \\
0.3 & - & 0.300 & - & \\
\hline \hline
\end{tabular}

HAO code differ slightly from the published results, ${ }^{10}$ although the parameters in the HAO algorithm are the same. It is likely that the published results were generated with an earlier version of the HAO code. The qualitative trends remain the same.)

In Ref. 10, the controllability canonical form is assessed as poorly conditioned because of the small minimum step size. However, Table 1 indicates that the static gain formulation in $\mathrm{H} 2 \mathrm{HOM}$ yields a substantial improvement in conditioning along the homotopy path over the HAO implementation of the canonical compensator. Although the static gain formulation is better conditioned for this example problem, this may not be the case in general. An even more significant benefit of this formulation is the straightforward extension to the $H_{\infty}$ and mixed $H_{2} / H_{\infty}$ problems.

Mixed $H_{2} / H_{\infty}$ Case

One-InputTwo-Output Case

The seminal paper dealing with mixed $H_{2} / H_{\infty}$ design addressed the case where $w=w_{p}$ in Eq. (37) with results from the four disk problem given for the full-order case. In this section, the oneinput/two-output case will be repeated with the FOCUS algorithm as well as a two-input/two-output case with full- and fixed-order compensators to demonstrate the capabilities of the homotopy algorithm.

Table 2 presents a comparison of the results from the FOCUS algorithm and the results published in Ref. 4. In Table 2, the BH subscript indicates results from Bemstein and Haddad ${ }^{4}$ and the $F$ subscript indicates results from FOCUS. Gaps in the columns of Table 2 denoted BH correspond to values of $\gamma$ for which results were not published. The absence of data generated by FOCUS for $\gamma=2$ and 1.5 is due to the fact that $\gamma$ is larger than the maximum $\infty$ norm, as described in the next section. A key distinction between the formulations of the mixed $H_{2} / H_{\infty}$ optimization problem used in this paper and that of Ref. 4 is that whereas their formulation minimizes an overbound on the $H_{2}$ norm, the formulation utilized in FOCUS minimizes the actual $\boldsymbol{H}_{2}$ norm. Consequently, as shown in Fig. 2, the FOCUS algorithm results in smaller $H_{2}$ norms for a given $\gamma$ with the gap in the $\infty$ norm overbound smaller than the Ref. 4 results in the meaningful region for $\gamma$, as described in the next section. Whereas $\gamma=0.52$ was the smallest $\gamma$ value reported in Ref. $4, \gamma$ values of $0.5,0.4$, and 0.3 are utilized with FOCUS, as indicated in Table 2. This example also demonstrates the synthesis of fixed-order mixed controllers with singular $H_{\infty}$ constraints. In practice, compensators representing the extreme values of $\gamma$ or $\lambda$ typically will not be used because either performance or robustness would be severely diminished. A compromise value in the elbow

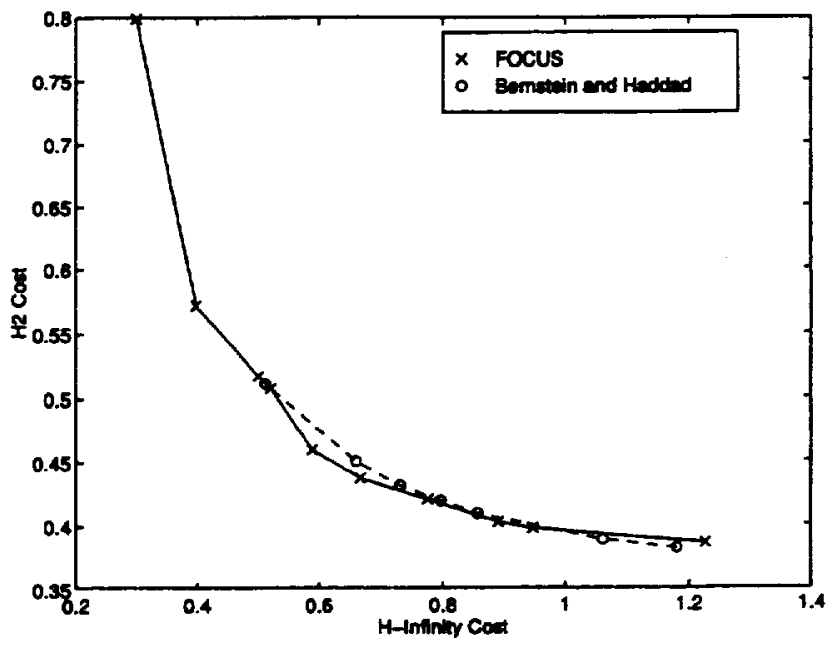

Fig. $2 H_{\infty}$ vs $H_{2}$ cost for single-input/multi-output example.

of Fig. 2 typically would be chosen. Although the FOCUS results in Table 2 are not significantly better than the results of Ref. 4, the example demonstrates that the FOCUS code performs satisfactorily and lessens the gap between the overbound and the $\infty$ norm.

\section{Discussion}

This example serves to illustrate some interesting features of the mixed $H_{2} / H_{\infty}$ formulation implemented in FOCUS, as well as distinctions from other formulations. The formulation implemented in FOCUS is a method for generating suboptimal $H_{\infty}$ controllers of fixed order that are subject to an $H_{2}$ constraint. The cost functional for the mixed $H_{2} / H_{\infty}$ problem can be written as

$$
J_{\operatorname{mix}}=J_{\infty}+\lambda J_{2}
$$

where

$$
\begin{gathered}
J_{\infty}=\operatorname{tr}\left\{Q_{\infty} \tilde{B} \tilde{B}^{T}\right\} \\
J_{2}=\operatorname{tr}\left\{X \tilde{C}_{p}^{T} \tilde{C}_{p}\right\}
\end{gathered}
$$

subject to the corresponding Riccati and Lyapunov equations. The resulting Lagrangian, also given by $\mathrm{Eq}$. (48), is

$$
\begin{aligned}
\mathcal{L} & =\operatorname{tr}\left\{Q_{\infty} \tilde{B} \tilde{B}^{T}+\left(\bar{A}^{T} Q_{\infty}+Q_{\infty} \bar{A}+\bar{C}^{T} \bar{C}+\gamma^{-2} Q_{\infty} \bar{B} \tilde{B}^{T} Q_{\infty}\right) L\right. \\
& \left.+\lambda X \bar{C}_{p}^{T} \bar{C}_{p}+\left(\bar{A} X+X \tilde{A}^{T}+\tilde{B}_{p} \tilde{B}_{p}^{T}\right) L_{p}\right\}
\end{aligned}
$$

Minimization of $J_{\infty}$ results in an $H_{\infty}$ controller with an upper bound on the $\infty$ norm given by the $\infty$ norm of the $H_{2}$ controller (as $\gamma \rightarrow \infty$, the $H_{2}$ compensator for $T_{z w}$ is recovered by minimizing $\left.J_{\infty}\right)$. By successively lowering gamma, the minimum $H_{\infty}$ norm controller for $T_{z w}$ is obtained. Minimization of $J_{2}$ results in the optimal $H_{2}$ compensator for $T_{z p w}$. Thus, when nonzero $\lambda$ is used in $J_{\text {mix }}$, the $H_{\infty}$ cost functional imposes an additional constraint on the $H_{2}$ norm of $T_{z p w}$, and for large $\gamma$, the necessary conditions for the mixed problem yield the simultaneous solution of two $\mathrm{H}_{2}$ problems. By increasing the $H_{2}$ weight, $\lambda$, for a fixed $\gamma,\left\|T_{z p w}\right\|_{2}$ is reduced while $\left\|T_{z w}\right\|_{\infty}$ approaches the gamma overbound. At that point, the minimum $H_{2}$ controller for $T_{z p w}$ such that $\left\|T_{z w}\right\|_{\infty} \leq \gamma$ is obtained.

For this example problem, the optimal $\mathrm{H}_{2}$ controller for $T_{z p w}$ results in $\left\|T_{z w}\right\|_{\infty}=1.392$ and $\left\|T_{z w}\right\|_{2}=0.3786$. The $\gamma$ values larger than 1.392 are not meaningful for our formulation because the optimal $\mathrm{H}_{2}$ compensator for $T_{z p w}$ satisfies the $\gamma$ constraint on $\left\|T_{z w}\right\|_{\infty}$. In that case, the $H_{\infty}$ norm constraint is inactive in the mixed norm optimization. However, since the Ref. 4 formulation seeks to minimize the $H_{2}$ norm overbound, it is possible for their formulation to generate meaningful solutions for $\gamma>1.392$. When using FOCUS, the first step should be to establish an upper bound on $\gamma$ by computing $\left\|T_{2 w}\right\|_{\infty}$ resulting from the optimal $\mathrm{H}_{2}$ compensator for $T_{z p w}$. 
Table 3 Comparison of two-input/two-output results

\begin{tabular}{lcccc}
\hline \hline$\gamma$ & $\left\|T_{z w}\right\|_{\infty, 8}$ & $\left\|T_{z p w p}\right\|_{2.8}$ & $\left\|T_{z w}\right\|_{\infty .4}$ & $\left\|T_{z p w p}\right\|_{2.4}$ \\
\hline 2 & $\overline{1.304}$ & $\overline{0.446}$ & 1.483 & 0.421 \\
1.5 & 1.000 & 0.500 & 1.000 & 0.437 \\
1.0 & 1.000 & 0.900 & 0.596 \\
0.9 & 0.900 & 0.529 & 0.800 & 0.587 \\
0.8 & 0.789 & 0.560 & 0.700 & 0.640 \\
0.7 & 0.694 & 0.652 & 0.600 & 0.952 \\
0.6 & 0.597 & 0.799 & 0.500 & 1.180 \\
0.5 & 0.500 & 3.842 & - & - \\
0.4 & 0.400 & 8.281 & - & - \\
0.3 & 0.300 & 24.84 & - & \\
\hline \hline
\end{tabular}

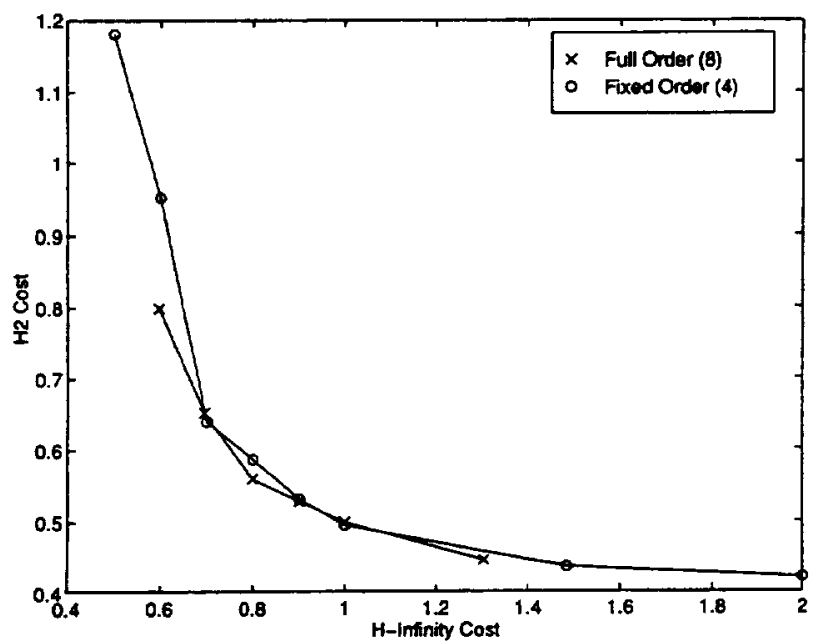

Fig. $3 H_{\infty}$ vs $H_{2}$ cost for MIMO example.

\section{Two-Inpur/Two-Output Reduced-Order Case}

To demonstrate the capability of the homotopy algorithm to synthesize fixed-order compensators for the two-input/two-output case, the four disk example was modified by creating a fictitious disturbance input for the $H_{\infty}$ minimization subproblem. The new coefficient matrices are given by $B_{1}=2 \times B_{p}$ and $D_{21}=2 \times D_{2 p}$. Additionally, a small penalty was placed on the control in the $H_{\infty}$ subproblem $\left(D_{12}=\left[\begin{array}{ll}0 & 0.001\end{array}\right]^{T}\right)$. Table 3 shows the results of the comparison for the full- and fixed-order two-input/two-output case. Figure 3 plots the intermediate values of Table 3 and illustrates the cost trade (extreme values are removed to preserve scale). In Table 3 , the subscript 8 indicates the full-order design and the subscript 4 indicates the fixed-order design. In general, full-order compensator synthesis is more taxing on the numerical algorithm due to the larger number of parameters to be optimized, which is evident in both Table 3 and Fig. 3. The fixed-order design is able to achieve smaller $\mathrm{H}_{2}$ norms at the smaller $\gamma$ values. Also, the $\infty$ norms for the larger values of $\gamma$ are closer to the overbound with the fixed-order designs. The reduced number of parameters to be optimized results in more accurate solution of the minimization problem. The closeness of the $\mathrm{H}_{2} / \mathrm{H}_{\infty}$ cost curves in Fig. 3 indicates that the fourth-order compensator closely approximates the performance/robustness tradeoff of an eighth-order compensator except at the low end of the attainable $H_{\infty}$ norm values. The infinity norm of $T_{z w}$ resulting from closing the loop with the full-order optimal $\mathrm{H}_{2}$ controller for $T_{2 p w p}$ is 1.845 , so that the full-order case with $\gamma=2$ was not a meaningful solution. However, a meaningful solution for $\gamma=2$ was achieved with the fixed-order compensator.

\section{Conclusions}

A homotopy algorithm is developed to synthesize fixed-order $H_{2}, H_{\infty}$, and mixed $H_{2} / H_{\infty}$ compensators employing a controller canonical form, and a representative flexible structure is used to demonstrate the numerical results. Fixed-order mixed $\mathrm{H}_{2} / \mathrm{H}_{\infty}$ compensators are synthesized for the case of one disturbance input set and two outputs sets, as well as the case of two disturbance input sets, two output sets. These numerical results indicate that for the example presented, the static output feedback formulation using the controller canonical form is a more efficient means of synthesizing dynamic compensators than employing a general compensator architecture. The synthesized reduced-order compensators perform well when compared to full-order controllers. The fixed-order mixed $\mathrm{H}_{2} / \mathrm{H}_{\infty}$ formulation is shown to offer improved performance over standard $H_{\infty}$ compensators by minimizing the $H_{2}$ norm while removing (or reducing) the gap between the actual $H_{\infty}$ norm and the gamma overbound.

\section{References}

'Bernstein, D. S., and Hyland, D. C., "Optimal Projection for Uncertain Systerns (OPUS): A Unified Theory of Reduced-Order Robust Control Design," Large Space Structures: Dynamics and Control, edited by S. N. Atluri and A. K. Amos, Springer-Verlag, New York, 1988, pp. 263302.

${ }^{2}$ Collins, E. G., Jr., Haddad, W. M., and Ying, S. S., "Reduced-Order Dynamic Compensation Using the Hyland-Bernstein Optimal Projection Equations," Journal of Guidance, Control, and Dynamics, Vol. 19, No. 2 , 1996, pp. 407-417.

${ }^{3}$ Ridgely, D. B., Canfield, R. A., Walker, D. E., and Smith, L. D., "The Fixed Order Mixed $\mathrm{H}_{2} / \mathrm{H}_{\infty}$ Control Problem: Development and Numerical Solution," The Intemational Joumal of Robust and Nonlinear Control (submitted for publication).

${ }^{4}$ Bemstein, D. S., and Haddad, W. M., "LQG Control with an $H_{\infty}$ Performance Bound: A Riccati Equation Approach," IEEE Transactions on Automatic Control, Vol. 34, March 1989, pp. 293-305.

${ }^{5}$ Rotea, M. A., and Khargonekar, P. P." " $H_{2}$-Optimal Control with an $H_{\infty}$ Constraint: The State Feedback Case," Automatica, Vol. 27, No. 2, 1991 , pp. 307-316.

${ }^{6}$ Jacques, D. R., Ridgely, D. B., Canfield, R. A., and Spillman, M. S., "A MATLAB ${ }^{\text {ni }}$ Toolbox for Fixed Order, Mixed-Norm Control Synthesis," Control Systems Magazine (to be published).

${ }^{7}$ Sweriduk, G. D., and Calise, A. J., "A Differential Game Approach to the Mixed $\mathrm{H}_{2} / \mathrm{H}_{\infty}$ Problem," Proceedings of the AIAA Guidance, Navigation, and Control Conference (Scottsdale, AZ), AIAA, Washington, DC, 1994, pp. 1072-1081 (AIAA Paper 94-3660).

${ }^{8}$ Whorton, M., Calise, A., and Buschek, H., "Development of Homotopy Algorithms for Fixed Order Mixed $H_{2} / H_{\infty}$ Controller Synthesis," NASA TP-3507, Sept. 1994.

${ }^{9}$ Davis, L. D., Collins, E. G., Jr., and Haddad, W. M., "Discrete-Time, Mixed-Norm $H_{2} / H_{\infty}$ Controller Synthesis," Optimal Control Application and Methods, Vol. 17, No. 2, 1996, pp. 107-122.

${ }^{10}$ Collins, E. G., Jr., Davis, L. D., and Richter, S. L., "Design of ReducedOrder $\mathrm{H}_{2}$ Optimal Controllers Using a Homotopy Algorithm," International Journal of Control, Vol. 61, No. 1, 1996, pp. 97-126.

${ }^{11}$ Mercadal, M., "Homotopy Approach to Optimal, Linear Quadratic, Fixed Architecture Compensation," Journal of Guidance, Control, and Dynamics, Vol. 14, No. 6, 1988, pp. 1224-1233.

${ }^{12}$ Kramer, F. S., and Calise, A. J., "Fixed-Order Dynamic Compensation for Multivariable Linear Systems," Journal of Guidance, Control, and Dynamics. Vol. 11, No. 1, 1988, pp. 80-85.

${ }^{13}$ Denery, D. G., "Identification of System Parameters from Input-Output Data with Application to Air Vehicles," Ph.D. Dissertation, Dept. of Applied Mechanics, Stanford Univ., Stanford, CA, May 1971.

${ }^{14}$ Buschek, H., "Synthesis of Fixed Order Controllers with Robustness to Mixed Real/Complex Uncertainies," Ph.D. Dissertation, School of Aerospace Engineering, Georgia Inst. of Technology, Atlanta, GA, Feb. 1995.

${ }_{15}$ Haddad, W. M., Collins, E. G., Jr., and Moser, R., "Structured Singular Value Controller Synthesis Using Constant D-Scales Without D-K Iteration," Intemational Journal of Control, Vol. 63, No. 4, 1996, pp. 813-830.

${ }^{16}$ Richter, S. L., and DeCarlo, R. A., "Continuation Methods: Theory and Applications," IEEE Transactions on Circuits and Systems, Vol. CAS-30, No. 6, 1983, pp. 347-352.

${ }^{17}$ Whorton, M., and Calise, A. J., "A Minimization Algorithm for IllConditioned Hessians," NASA TM (submitted for publication).

${ }^{18}$ Collins, E. G., Jr., Haddad, W.M., and Ying, S. S., "Nearly Nonminimal Linear Quadratic Gaussian Reduced-Order Control Design Initialization," Joumal of Guidance, Control, and Dynamics, Vol. 19, No. 1, 1996, pp. 259-262.

${ }^{19}$ Cannon, R. H., Jr., and Rosenthal, D. E., "Experiments in Control of Flexible Structures with Noncolocated Sensors and Actuators," Joumal of Guidance, Control, and Dynamics, Vol. 7, No. 5, 1984, pp. 546-553. 


